\title{
Time measurement technique for internal friction studies with a torsion pendulum
}

\author{
C N RAO and M K ASUNDI \\ Metallurgy Division, Bhabha Atomic Research Centre, Bombay 400 085, India \\ MS received 10 December 1982; revised 27 January 1983
}

\begin{abstract}
A new technique for measuring internal friction by the free decay method using time measurements with a torsion pendulum is described. It has been applied for the automation of measurements with a Kêt type pendulum. The method enables rapid and precise measurement of internal friction. Results of the grain boundary peak for aluminium are presented for illustration.
\end{abstract}

Keywords. Internal friction; torsion pendulum; automation of measurement; Kê type pendulum.

\section{Introduction}

The torsion pendulum is an extensively used device to study the low frequency internal friction of materials (Kê 1947; Ang and Wert 1954). Two basic methods, the free decay and the constant amplitude methods, are generally employed to abtain the internal friction values from the pendulum apparatus.

In the free decay method, the amplitude of free vibrations is measured over a number of cycles and the internal friction $Q^{-1}$ is obtained in terms of the damping $\delta$ by the relation

$$
Q^{-1}=\delta / \pi=(1 / n \pi) \ln \left(A_{i} / A_{i}+n\right)
$$

where $A_{i}$ is the amplitude of the $i$ th cycle and $n$ is the number of cycles over which the measurement is made.

In the constant amplitude method, the oscillations of the pendulum are maintained at a constant amplitude by supplying a certain energy $\Delta W$ in each cycle and $Q^{-1}$ is obtained by the relation

$$
Q^{-1}=\Delta W / 2 \pi W
$$

where $W$ is the total energy of the pendulum.

To carry out extensive studies of internal friction over a large range of temperatures and for a number of frequencies, automation of the pendulum apparatus is very helpful. Several versions of automation have been reported for the free decay method 
(de Morton et al 1963; Bays and Grandchamp 1970; Smith et al 1970; Ritchie et al 1973; Yoshida et al 1981) as well as for the constant amplitude method (Salvi et al 1965; Perez et al 1967; Butera and Craig 1966; Barrow and Szkopiak 1972; Brozel and Leak 1978).

Almost all the techniques using the free decay method depend on sensing the amplitude of oscillation to compute the damping. In addition to the requirement of finding the amplitude, both low as well as very large values of damping impose some practical problems. If the damping is large, the amplitude decays too quickly causing inaccuracy in the measurement. If it is too small, the measurement has to be carried out over a large number of cycles to get a measurable change of amplitude. In the measurement of the temperature variation of internal friction, this would require a very slow rate of heating to enable measurement at a particular temperature. If the measurements could be carried out over a few cycles only, it will be possible to use faster heating rates governed solely by the need of uniform sample temperature. The procedure given by Yoshida et al (1981) achieves this by digitization and Fourier analysis of the oscillogram of the vibrations, but the method requires an on line computer for data processing.

This paper describes a technique for the determination of internal friction by the free decay method using time measurements over a few cycles which leads to automation quite easily.

\section{Principle of measurement}

Figure 1 shows the operation of the pendulum apparatus. The electromagnets $E$ are used to start the oscillations of the pendulum. A focussed beam of light reflected from the mirror $M$ of the pendulum falls on two photo-detectors $D_{1}$ and $D_{2}$ placed at a large distance from the pendulum, and provides marker pulses to carry out the necessary time measurements (figure 2). From the time measurements, two different procedures can be applied for evaluating the parameters of the pendulum. The first one is based on finding the velocity at the equilibrium position and the second on an iterative least squares procedure using the time measurements from either of the detectors.

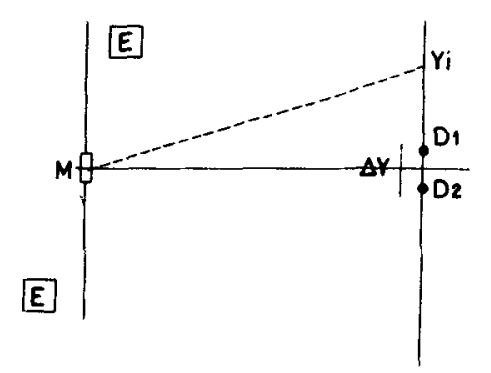

Figure 1. Diagram of pendulum operation. (M, mirror; $D_{1}$ and $D_{2}$, photo-detectors; E-electromagnet for starting torsional vibrations) 


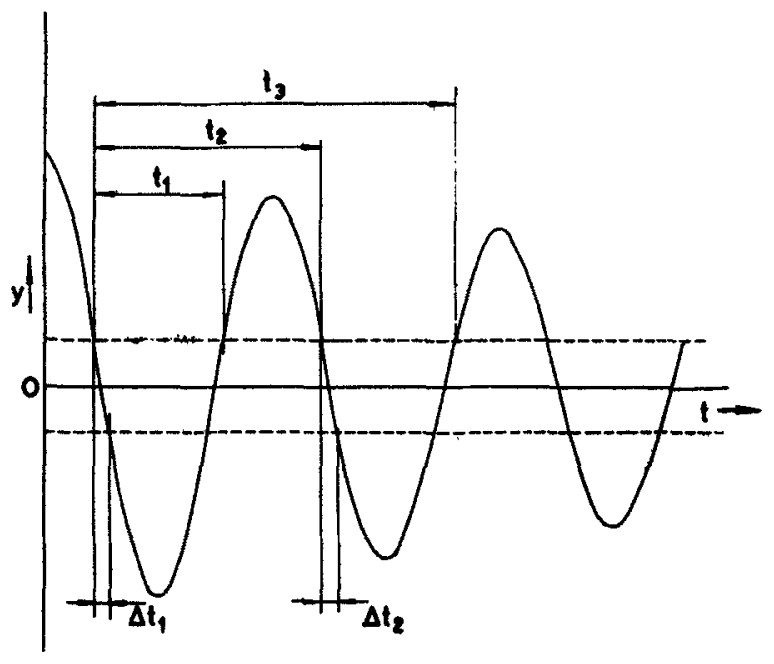

Figure 2. Time intervals needed for computing the damping.

\subsection{Velocity measurement method}

For free oscillations of the pendulum, the displacement $Y$ (of the light spot in the plane of the detectors) at a given time $t$, measured from the instant of the amplitude $Y_{i}$, can be written as

$$
Y=Y_{i} \exp (-\delta t) \cos \omega t
$$

where $\omega$ is the angular frequency.

If $D_{1}$ and $D_{2}$ are placed symmetrically about the equilibrium position at a small distance $\Delta y$ apart such that $\Delta y \ll Y_{i}$, the velocity $V_{i}$ of the light spot as it crosses the equilibrium position can be written with good approximation as

$$
V_{i}=\left(\Delta Y / \Delta t_{i} \simeq Y_{i} \omega(1-\pi \delta / 2 \omega)\right.
$$

where $\Delta t_{i}$ is the time taken for the light spot to traverse from the first to the second detector. The amplitude $Y_{i}$ can therefore be obtained as

$$
Y_{i}=\Delta Y /\left[\Delta t_{i} \omega(1-\pi \delta / 2 \omega)\right]
$$

By measuring the time intervals at which the light crosses the detectors in the same direction in two successive cycles, the log decrement is obtained as

$$
\delta=\ln \left(\Delta t_{i+1} / \Delta t_{i}\right)
$$

Also, the values of the angular frequency $\omega$ and the initial amplitude $Y_{i}$ can be deduced with sufficient accuracy from the time measurements. Using fast response photo- 
detectors and a sharply focussed light spot, the $\Delta t$ values can be measured correct to a fraction of a $\mu \mathrm{sec}$. Taking typical values of $\omega=2 \pi, \Delta y=1 \mathrm{~cm}, Y_{i}=5 \Delta y$ and an error of $0.2 \mu \mathrm{sec}$ in $\Delta t$, the precision in the measurement of $\delta$ will be $0.006 \%$ and $6 \%$ at $10^{-1}$ and $10^{-4}$ respectively. Thus, the error due to the technique of measurement is quite small compared to other possible contributions like that due to mechanical instabilities in the apparatus etc.

The method requires the detectors to be placed symmetrically about the equilibrium position of the light spot. However, in actual practice, as the sample temperature is increased, there will be some shift in the equilibrium position due to untwisting of the specimen. Careful annealing and fixing of the sample can reduce the effect; but it can be significant when studying deformed metals. Hence for the automation of the experiment by this method, provision should be made for adjusting the position of the detectors during the experiment. A second limitation will be due to the requirement of $\Delta y \ll Y_{i}$ which may not be valid over several cycles if the damping is too large.

In order to avoid the limitations of the above two factors, the following iterative method can be employed to derive the parameters of the torsion pendulum from the time measurements.

\subsection{Iterative least squares method}

If we measure time from the instant the light spot crosses one of the detectors and denote the time interval as $t_{i}$ for the crossing of the light spot over the same detector (figure 2), then for these $t_{i}$ values the displacement of the pendulum will have some constant values. If the initial phase is $\phi$, this constant displacement is given by

$$
Y_{0} \exp (-\delta \phi / \omega) \cos \phi=Y_{0} \exp (-\delta \phi / \omega) \exp \left(-\delta t_{i}\right) \cos \left(\omega t_{i}+\phi\right)
$$

where $\mathrm{Y}_{0}$ is the amplitude at the start of the free decay. Denoting $\tan \phi$ by $A$, the above equation can be re-written as

$$
F_{i}=\cos \omega t_{i}-A \sin \omega t_{i}-\exp \left(\delta t_{i}\right)
$$

Knowing-approximate values of $\omega, A$ and $\delta$ (which can be determined by a procedure similar to the first one described earlier) more precise values of these parameters can be computed by an iterative method. For this purpose, let the true values be denoted by $\omega_{0}=\omega+\Delta \omega, A_{0}=A+\Delta A, \delta_{0}=\delta+\Delta d$ and $F_{i 0}=F_{i}+\Delta F_{i}$.

Expanding in a Taylor's series and retaining the first order terms, we obtain

$$
-\Delta F_{i}=\Delta A \sin \omega t_{i}+\Delta \omega t_{i}\left(\sin \omega t_{i}+A \cos \omega t_{i}\right)+\Delta \delta t_{i} \exp \left(\delta t_{i}\right)
$$

Using the $t_{i}$ values over two or more cycles, this relation gives a set of linear equations that can be solved by a least squares procedure to get the correction terms. The corrected set forms the basis for the next cycle of iteration and so on till the values are obtained with sufficient precision. Time measurements with respect 
to the two detectors $D_{1}$ and $D_{2}$ give two sets of linear equations which can be solved seperately to obtain $\omega$ and $\delta$ without involving the distance between the detectors.

The drift of the zero position of the pendulum is significant only over a long period of time corresponding to a temperature change of several degrees. Hence it will not influence the time measurements over a few cycles of the pendulum (which will be a few seconds for the frequencies normally used). In addition, it is not necessary to have the detectors exactly symmetrical about the equilibrium position. In the rare event of one of the detectors coinciding with the equilibrium position, the $A$ value in (8) becomes undefined; in such a case only one set of $t_{i}$ will be available for computing the parameters of the pendulum.

The limitation due to large damping values is not at all important here so long as the amplitude remains large enough to allow the light spot to cross the detectors.

\section{Implementation of the technique}

The technique of measurement presented above had been used to automate internal friction measurements with a $\mathrm{Ke}$ type torsion pendulum. A He-Ne laser source was focussed on to the mirror of the pendulum to produce a sharp rectangular spot of $10 \times 1 \mathrm{~mm}$ size at the detectors placed $5 \mathrm{~m}$ away from the mirror. Two photodiodes placed $1 \mathrm{~cm}$ apart symmetrically about the equilibrium position served as detectors to provide marker pulses for measuring time intervals by means of a 10 $\mathrm{MHz}$ clock. The pendulum was excited to obtain an initial deflection of the light spot of about 5-6 cm at the location of the detectors and the time measurements were carried out over four cycles.

A sample of pure aluminium wire $15 \mathrm{~cm}$ long and $0.1 \mathrm{~cm}$ diameter was used in the torsion pendulum which gave a frequency of $0.95 \mathrm{~Hz}$ at $26^{\circ} \mathrm{C}$. The sample was heated at the rate of $5^{\circ} \mathrm{C} / \mathrm{min}$ and internal friction measurements were carried out at about $20^{\circ} \mathrm{C}$ at intervals in the range $100-400^{\circ} \mathrm{C}$. During heating of the sample, the zero position was found to drift by several $\mathrm{mm}$. For the set of readings used to deduce $\delta$ by the first method, the position of the detector system was adjusted whenever the zero drift exceeded one $\mathrm{mm}$. The $Q^{-1}$ values computed by both the methods were in agreement up to three significant digits.

The grain boundary relaxation peak obtained from the sample is given in figure 3. The peak is in general agreement with the results for aluminium (Kê 1947).

To check the reproductibility, one of the readings (at $210^{\circ} \mathrm{C}$ ) was repeated 20 times. The average $Q^{-1}$ value obtained was $19.69^{-} \times 10^{-3}$ with a standard deviation of $0.05 \times 10^{-3}$

The accuracy of internal friction measurements depends on the damping. Parasitic vibrations of the pendulum apparatus usually set a limit to the lowest values of the damping that can be measured. The present technique enables precise measurements of low damping values using only a few cycles of the pendulum. In this respect it stands better than the conventional ones based on sensing the amplitude of vibration for which measurement has to be carried out over a large number of cycles to determine low damping values. 


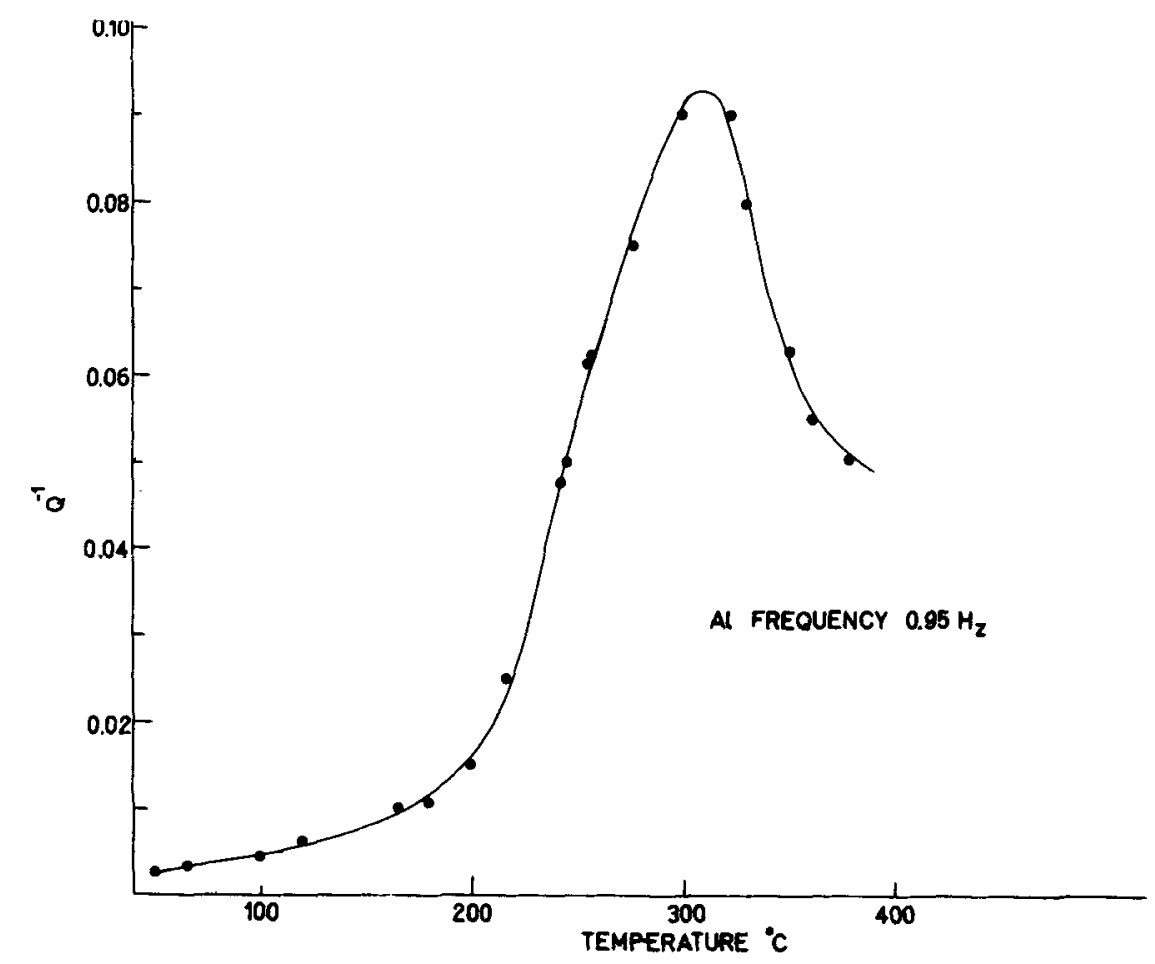

Figure 3. Typical results of the variation of damping with temperature for aluminium.

\section{Conclusions}

The results obtained clearly show that the time measurement technique described here can be used effectively to automate low frequency internal friction measurements by the free decay method. A microprocessor interfaced with a calculator can easily provide the necessary computations.

The velocity measurement method requires the amplitude to be large compared to the distance between the detectors. Also it is necessary to adjust the position of the detectors to compensate for the drift of the equilibrium position. The iterative method is free from the above limitations. Since the time measurements are required over only a few cycles of oscillation of the pendulum, the technique enables rapid and precise measurement of internal friction.

\section{References}

Ang C Y and Wert C 1954 J. Appl. Phys. 251061

Barrow D E and Szkopiak Z C 1972 J. Phys. E 5915 
Bays B and Grandchamp P A 1970 Rev. Phys. Appl. 5327

Brozel M R and Leak G M 1978 J. Phys. E 1153

Butera R A and Craig R S 1966 Rev. Sci. Instrum. 37401

Kê T S 1947 Phys. Rev. 71533

de Morton M E, Lott S A and Stainsby K J 1963 J. Sci. Instrum. 40441

Perez J, Delorme J, Peguin P and Gobin P 1967 J. Sci. Instrum. 44169

Ritchie I G, Saltvold J R, Schmidt H K and Sprungman K W 1973 J. Phys. E. 6341

Salvi A, Dautreppe D and Friess E 1965 Reo. Sci. Instrum. 36198

Smith D L, Winiecki A L and Lee R H 1970 J. Phys. E 3715

Yoshida I, Sugai T, Tani S, Motegi M, Minamida K and Hayakawa H 1981 J. Phys. E 141201 